\title{
Seeking Observable Imprints of Small-Scale Structure on the Properties of Dark Matter Haloes
}

\author{
C. Power ${ }^{1,2}$ \\ ${ }^{1}$ International Centre for Radio Astronomy Research, University of Western Australia, 35 Stirling Highway, Crawley WA 6009, Australia \\ ${ }^{2}$ Email: chris.power@icrar.org
}

(Received August 27, 2013; AccePted September 6, 2013; Online Publication October 14, 2013)

\begin{abstract}
The characteristic prediction of the Cold Dark Matter (CDM) model of cosmological structure formation is that the Universe should contain a wealth of small-scale structure-low-mass dark matter haloes and subhaloes. However, galaxy formation is inefficient in their shallow potential wells and so we expect these low-mass haloes and subhaloes to be dark. Can we tell the difference between a Universe in which these low-mass haloes are present but dark and one in which they never formed, thereby providing a robust test of the CDM model? We address this question using cosmological $\mathrm{N}$-body simulations to examine how properties of low-mass haloes that are potentially accessible to observation, such as their spatial clustering, rate of accretions and mergers onto massive galaxies, and the angular momentum content of massive galaxies, differ between a fiducial $\Lambda \mathrm{CDM}$ model and dark matter models in which low-mass halo formation is suppressed. Adopting an effective cut-off mass scale $M_{\text {cut }}$ below which small-scale power is suppressed in the initial conditions, we study dark matter models in which $M_{\text {cut }}$ varies between $5 \times 10^{9} h^{-1} \mathbf{M}_{\odot}$ and $10^{11} h^{-1} \mathbf{M}_{\odot}$, equivalent to the host haloes of dwarf and low-mass galaxies. Our results show that both the clustering strength of low-mass haloes around galaxy-mass primaries and the rate at which they merge with these primaries are sensitive to the assumed value of $M_{\text {cut }}$; in contrast, suppressing low-mass halo formation has little influence on the angular momentum content of galaxy-mass haloes - it is the quiescence or violence of a halo's assembly history that has a more marked effect. However, we expect that measuring the effect on spatial clustering or the merger rate is likely to be observationally difficult for realistic values of $M_{\text {cut }}$, and so isolating the effect of this small-scale structure would appear to be remarkably difficult to detect, at least in the present day Universe.
\end{abstract}

Keywords: cosmology: theory - dark matter - galaxies: formation - galaxies: haloes - large-scale structure of Universe

\section{INTRODUCTION}

One of the key questions facing fundamental physics and cosmology at the turn of the 21 st century concerns the nature of the dark matter. Approximately $80 \%$ of the matter content of the Universe appears to be in the form of exotic, nonbaryonic dark matter (cf. Planck Collaboration et al. 2013) whose clustering is believed to play a crucial role in the formation and subsequent evolution of galaxies (e.g. White \& Rees 1978; White \& Frenk 1991). This non-baryonic dark matter is widely assumed to be cold-that is, dark matter particles were non-relativistic at the time of decouplingand collisionless, and these properties of Cold Dark Matter (hereafter CDM) lead to a number of fundamental consequences. The first of these is that dark matter haloes have central density cusps (cf. Tremaine \& Gunn 1979; Moore 1994); the second is that the halo mass function-the number of haloes of mass $M$ per unit mass per unit comoving volume - increases with decreasing mass as $M^{-\alpha}$ where $\alpha \sim$ 2.0 (cf. Table 3 of Murray et al. 2013a) down to masses that could be as small as $\sim 10^{-6} \mathrm{M}_{\odot}$ (cf. Green et al. 2004).

Based on the results of cosmological $N$-body simulations, cuspy haloes and an abundance of small-scale structurelow-mass haloes and subhaloes-are now well established as robust predictions of the CDM model (e.g. Springel et al. 2008). If we consider a Universe in which low-mass halo and subhalo formation is suppressed, as in Warm Dark Matter (hereafter WDM) models, we find that haloes can form cores (albeit small ones; cf. Villaescusa-Navarro \& Dalal 2011) but they are likely to remain cuspy for plausible WDM particle masses $\left(m_{\mathrm{WDM}} \gtrsim 0.5-2 \mathrm{keV}\right)$, even when free streaming is accounted for (e.g. Colín et al. 2008; Macciò et al. 2012). In contrast, we expect the abundance and clustering of low-mass haloes to be suppressed in WDM models (e.g. Dunstan et al. 2011; Smith \& Markovic 2011; Schneider et al. 2013; Benson et al. 2013; Pacucci et al. 2013), and so it can be argued that it 
is the abundance of small-scale structure, rather than central density cusps, that is the defining characteristic of the CDM model $^{1}$.

However, we expect few of these low-mass haloes to host galaxies because galaxy formation will be inefficient in their shallow potential wells (e.g. Dekel \& Silk 1986; Efstathiou 1992; Thoul \& Weinberg 1996; Benson et al. 2002). For example, supernovae (e.g. Dekel \& Silk 1986) and photoionising sources (e.g. Benson et al. 2002; Cantalupo 2010) can quench galaxy formation in low-mass haloes, while the likelihood that a low-mass halo hosts a satellite galaxy appears to be stochastic (cf. Boylan-Kolchin et al. 2012; Garrison-Kimmel et al. 2013), suggesting that the process is highly sensitive to details of a galaxy's assembly history (i.e. environment, gas accretion and star formation history, etc...). This raises the question, if low-mass haloes and subhaloes remain dark because galaxy formation is inefficient on these mass scales, how can we tell the difference between a Universe in which small-scale structure is present but dark and one in which its formation is suppressed, as in WDM models?

In this paper, we use the results of cosmological $N$-body simulations to address this question, comparing systematically dark matter halo properties in a fiducial $\Lambda \mathrm{CDM}$ cosmology and in $\Lambda \mathrm{WDM}$-like dark matter models, in which low-mass halo formation is suppressed by truncating the $\Lambda \mathrm{CDM}$ power spectrum on small scales. Numerous studies have investigated the halo mass function in WDM models (recent examples include Schneider et al. 2012, 2013; Pacucci et al. 2013; Benson et al. 2013) and associated issues arising from discreteness effects in such simulations (e.g. Wang \& White 2007; Schneider et al. 2013; Angulo et al. 2013; Hahn et al. 2013), but we note that direct measurement of the halo mass function observationally is fraught with difficulty (cf. Murray et al. 2013b). For this reason, we focus on three measures of the halo population that are potentially accessible to observation-(i) the spatial clustering of low-mass haloes around galaxy- and group-mass haloes $\left(10^{11} h^{-1} \mathrm{M}_{\odot} \lesssim M_{\mathrm{vir}} \lesssim\right.$ $10^{13} h^{-1} \mathrm{M}_{\odot}$ ); (ii) the rate at which these haloes assemble their mass and at which they experience mergers; and (iii) their angular momentum content. Although we analyse properties of the halo population, we reason that they provide a baseline for trends that we observe in the galaxy population.

We choose the cut-off mass $M_{\text {cut }}$, the mass scale below which halo formation is suppressed, to vary between $5 \times 10^{9} h^{-1} \mathrm{M}_{\odot} \lesssim M_{\text {cut }} \leqslant 10^{11} h^{-1} \mathrm{M}_{\odot}$. These values of $M_{\text {cut }}$ are unrealistic in the sense that they are too large to be consistent with observational constraints (see, for example, the review

\footnotetext{
${ }^{1}$ There is a caveat here-we have assumed implicitly that dark matter is collisionless. Recent papers have revived the possibility that dark matter is self-interacting (e.g. Loeb \& Weiner 2011; Vogelsberger et al. 2012; Peter et al. 2013; Rocha et al. 2013), an idea that was first explored systematically using simulations in the early 2000s (see, for example Yoshida et al. 2000; Davé et al. 2001; Colín et al. 2002). This recent work has shown that it is possible to form small cores $\left(r_{\text {core }} \simeq 1 \mathrm{kpc}\right)$ in Milky Way mass galaxies (Loeb \& Weiner 2011) while leaving the abundance and radial distribution of dark matter subhaloes unchanged from the prediction of the CDM model (e.g. Vogelsberger et al. 2012; Rocha et al. 2013).
}

of Primack 2009, assuming corresponding filtering masses from Bode et al. 2001) but they allow us to experiment with the consequences of progressively more aggressive truncations of the initial power spectrum on the properties of haloes with $M \gtrsim 10^{11} \mathrm{M}_{\odot}$.

The structure of this paper is as follows. In Section 2, we describe our simulations, detailing how we set them up, and summarising our approach to constructing merger trees and halo sample selection. In Section 3, we focus on the spatial clustering of haloes and demonstrate that the clustering strength of low-mass haloes is suppressed relative to the $\Lambda \mathrm{CDM}$ model in our truncated models. We show how this suppression in clustering impacts the number and frequency of minor mergers (Section 4) and we explore measures of halo angular momentum and spin (Section 5). Finally, in Section 6 we summarise our results, assessing their implications for developing robust astrophysical tests of the nature of the dark matter.

\section{THE SIMULATIONS}

We have run a sequence of cosmological $N$-body simulations that follow the formation and evolution of dark matter haloes in a box of side $20 h^{-1} \mathrm{Mpc}$ from a starting redshift of $z=$ 100 to $z=0$. For each run, we assume a flat cosmology with a dark energy term, and for convenience we adopt the cosmological parameters of Spergel (2007) — matter and dark energy density parameters of $\Omega_{\mathrm{m}}=0.24$ and $\Omega_{\Lambda}=0.76$, a dimensionless Hubble parameter of $h=0.73$, a normalisation of $\sigma_{8}=0.74$, and a primordial spectral index of $n_{\text {spec }}=0.95$. Each simulation volume contains $256^{3}$ equal-mass particles, which for the adopted cosmological parameters gives particle masses of $m_{\mathrm{p}}=3.176 \times 10^{7} h^{-1} \mathrm{M}_{\odot}$.

The respective runs differ in the spatial scale below which small-scale power in the initial conditions is suppressed. We generate a single realisation of the $\Lambda \mathrm{CDM}$ power spectrum appropriate for our choice of cosmological parameters and in the case of the truncated models we introduce a sharp cut-off in the $\Lambda \mathrm{CDM}$ power spectrum at progressively larger spatial scales. This cut-off spatial scale is set by the mass scale below which we wish to suppress halo formation. Details about the truncated models are given in the next section.

All of our simulations were run using the parallel TreePM code GADGET2 (Springel 2005) with a constant comoving gravitational softening $\epsilon=1.5 h^{-1} \mathrm{kpc}$ and individual and adaptive particle time-steps. These were assigned according to the criterion $\Delta t=\eta \sqrt{\epsilon / a}$, where $a$ is the magnitude of a particle's gravitational acceleration and $\eta=0.05$ determines the accuracy of the time integration.

\subsection{Truncated dark matter models}

\subsubsection{Truncating the initial power spectrum}

We are interested in models in which small-scale power is suppressed at early times. Physically suppression arises 
because dark matter free streams, which acts as a damping mechanism to wash out primordial density perturbations and to introduce a cut-off in the linear matter power spectrum. If the dark matter particle is a thermal relic, the spatial scale at which this cut-off occurs can be calculated (cf. Bergström 2000). The free streaming scale $\lambda_{\mathrm{fs}}$ can be expressed as

$$
\lambda_{\mathrm{fs}}=0.2\left(\Omega_{\mathrm{dm}} h^{2}\right)^{1 / 3}\left(\frac{m_{\mathrm{dm}}}{\mathrm{keV}}\right)^{-4 / 3} \mathrm{Mpc},
$$

where $m_{\mathrm{dm}}$ is the dark matter particle mass measured in $\mathrm{keV}$ and $\Omega_{\mathrm{dm}}$ is the dark matter density (cf. Boehm et al. 2005).

Provided $\lambda_{\mathrm{fs}}$ is small compared to the spatial scales we are interested in simulating, the power spectrum will differ little from the $\Lambda \mathrm{CDM}$ power spectrum (which itself may have a cut-off on comoving scales of order 1 pc; cf. Green et al. 2004). However, as $\lambda_{\mathrm{fs}}$ increases and approaches the scale that we wish to resolve, then it becomes necessary to determine how the power spectrum changes. The shape of the linear power spectrum for collisionless WDM models has been calculated by a number of authors (e.g. Bardeen et al. 1986; Bode et al. 2001), and it can be recovered from the CDM power spectrum by introducing an exponential cutoff at small scales. The larger $\lambda_{\mathrm{fs}}$, the larger the mass scale $\mathrm{M}_{\mathrm{fs}}$ below which structure formation is suppressed and the smaller the wave-number $k$ at which the WDM and CDM power spectra differ, although the relationship between $\lambda_{\mathrm{fs}}$ and $\mathrm{M}_{\mathrm{fs}}$ is sensitive to the precise nature of the WDM particle.

We do not wish to make any assumptions about the precise nature of the dark matter other than that it is collisionless and that low-mass halo formation is suppressed, and so we follow Moore et al. (1999) and truncate sharply the power spectrum at $k_{\text {cut }}$, suppressing power at wave-numbers $k \geq k_{\text {cut }}$. We choose $k_{\text {cut }}$ by identifying a mass scale $M_{\text {cut }}$ and estimating the comoving length scale $R_{\text {cut }}$,

$$
R_{\text {cut }}=\left(\frac{3 M_{\text {cut }}}{4 \pi} \frac{1}{\bar{\rho}}\right)^{1 / 3},
$$

where $\bar{\rho}$ is the mean density of the Universe.

\subsubsection{Modelling free streaming}

Similarly, we choose not to include the effect of free streaming in our initial conditions-partly because we wish to avoid assumptions about the precise nature of the dark matter, and partly for pragmatic reasons, which we now explain. In practice, free streaming is mimicked by assigning a random velocity component (typically drawn from a Fermi-Dirac distribution) to particles in addition to their velocities predicted by linear theory (cf. Klypin et al. 1993; Colín et al. 2008; Macciò et al. 2012). However, capturing this effect correctly in a $N$-body simulation is difficult-it can lead to an unphysical excess of small-scale power in the initial conditions if the simulation is started too early (see Figure 1 of Colín et al. 2008 for a nice illustration of this problem).

Precisely, how early is too early has yet to be properly quantified, but it will depend explicitly on dark matter particle mass-the lower the mass, the longer the free streaming
Table 1 Truncated models : simulation details.

\begin{tabular}{lccr}
\hline \hline Model & $\begin{array}{c}M_{\text {cut }} \\
10^{10} h^{-1} \mathrm{M}_{\odot}\end{array}$ & $\begin{array}{c}R_{\text {cut }} \\
h^{-1} \mathrm{Mpc}\end{array}$ & $\begin{array}{r}k_{\text {cut }} \\
h \mathrm{Mpc}^{-1}\end{array}$ \\
\hline A & 0.5 & 0.26 & 24.01 \\
B & 1.0 & 0.33 & 19.06 \\
C & 5.0 & 0.56 & 11.15 \\
D & 10.0 & 0.71 & 8.85 \\
\hline \hline
\end{tabular}

scale, and the larger the random velocity component required. If this exceeds the typical velocity predicted by linear theory, a population of spurious haloes forms (e.g. Klypin et al. 1993) that can exceed in number by factors of $\sim 10$ the population that forms when no random velocity component is included, as studied by Wang and White (2007). This is unlikely to be a problem for studying the mass profiles of haloes-for example, Colín et al. (2008) started their simulations at reasonably late times because the random velocity component damps away with decreasing redshift while the velocities predicted by linear theory increase-but it is not clear how much of a problem it will be for our study, in which we study quantities that depend on spatial correlations between haloes. For this reason, we do not include the effect of free streaming, deferring this to a forthcoming study on discreteness effects in WDM-like simulations (C. Power et al., in preparation).

\subsubsection{Generation of initial conditions}

We follow the standard procedure (e.g. Power et al. 2003) of generating a statistical realisation of the high redshift density field using the appropriate linear theory power spectrum, from which initial displacements and velocities are computed and imposed on a suitable uniform particle load; for this study we adopt an initial glass distribution (cf. White 1994). We use the Boltzmann code CMBFAST (Seljak \& Zaldarriaga 1996) to generate the CDM transfer function for our choice of cosmological parameters. This is convolved with the primordial power spectrum $\left(P(k) \propto k^{n}\right.$, where $n$ is the primordial spectral index) to obtain the appropriate $\Lambda \mathrm{CDM}$ power spectrum $P(k)$. To obtain a truncated model, we chop $P(k)$ sharply at $k_{\text {cut }}=2 \pi / R_{\text {cut }}$ (where $R_{\text {cut }}$ is given by Equation (2)) and thereby suppress power on scales $k \gtrsim k_{\text {cut }}$.

We consider five cases-a fiducial $\Lambda \mathrm{CDM}$ model and truncated models in which small-scale power is suppressed at masses below $M_{\text {cut }}=5 \times 10^{9}, 10^{10}, 5 \times 10^{10}$ and $10^{11} h^{-1} \mathrm{M}_{\odot}$, respectively. Note that the cut-off wave-number $k_{\text {cut }}$ is always less than the Nyquist frequency of the simulation, $k_{\mathrm{Ny}} \simeq 40 \mathrm{hpc}^{-1}$. Values for the cut-off masses and wavenumbers are given in Table 1.

\subsection{Halo identification and merger trees}

\subsubsection{Halo identification}

Groups are identified using AMIGA's Halo Finder (AHF) (cf. Knollmann \& Knebe 2009). AHF locates groups as peaks 
in an adaptively smoothed density field using a hierarchy of grids and a refinement criterion that is comparable to the force resolution of the simulation. Local potential minima are calculated for each of these peaks and the set of particles that are gravitationally bound to the peaks are identified as the groups that form our halo catalogue. Each halo in the catalogue is then processed, producing a range of structural and kinematic information.

We adopt the standard definition of a halo such that the virial mass is

$$
M_{\text {vir }}=4 \pi \rho_{\text {crit }} \Delta_{\text {vir }} r_{\text {vir }}^{3} / 3,
$$

where $\rho_{\text {crit }}=3 H^{2} / 8 \pi G$ is the critical density of the Universe and $r_{\text {vir }}$ is the virial radius, which defines the radial extent of the halo. The virial over-density criterion, $\Delta_{\text {vir }}$, is a multiple of the critical density, and corresponds to the mean over-density at the time of virialisation in the spherical collapse model (the simplest analytic model of halo formation; cf. Eke et al. 1996). In an Einstein-de Sitter Universe, $\Delta_{\mathrm{vir}} \simeq 178$, while in the favoured $\Lambda \mathrm{CDM}$ model $\Delta_{\mathrm{vir}} \simeq 92$ at $z=0$.

Defined in this way, the virial radius $r_{\text {vir }}$ provides a convenient albeit approximate boundary for a dark matter halo that can be estimated easily from simulation data. However, it is only approximate-haloes that form in cosmological simulations are relatively complex structures. They are generally aspherical (e.g. Bailin \& Steinmetz 2005) and asymmetric (e.g. Gao \& White 2006) with no simple boundary (e.g. Prada et al. 2006), and so defining an appropriate boundary is not straightforward. This presents difficulties when calculating, for example, a halo's angular momentum and its binding energy (cf. Łokas \& Mamon 2001). Material bound to the halo can lie outside of $r_{\text {vir }}$, and this will distort the angular momentum and binding energy one measures for the halo using only material from within $r_{\mathrm{vir}}$. This issue has been touched on by previous authors (e.g. Cole \& Lacey 1996; Łokas \& Mamon 2001; Shaw et al. 2006; Power et al. 2012 ) in the context of identifying when a halo is in virial equilibrium. In a similar vein, the angular momentum one measures using only material from within $r_{\text {vir }}$ will be biased. This is an important caveat that we need to bear in mind when discussing our analysis of halo angular momentum in Section 5.

\subsubsection{Halo merger trees}

Halo merger trees are constructed by linking halo particles at consecutive output times.

- For each pair of group catalogues constructed at consecutive output times $t_{1}$ and $t_{2}>t_{1}$, the 'ancestors' of 'descendant' groups are identified. For each descendent identified in the catalogue at the later time $t_{2}$, we sweep over its associated particles and locate every ancestor at the earlier time $t_{1}$ that contains a subset of these particles. A record of all ancestors at $t_{1}$ that con- tain particles associated with the descendent at $t_{2}$ is maintained.

- The ancestor at time $t_{1}$ that contains in excess of $f_{\text {prog }}$ of these particles and also contains the most bound particle of the descendent at $t_{2}$ is deemed the main progenitor. Typically $f_{\text {prog }}=0.5$, i.e. the main progenitor contains in excess of half the final mass.

Each group is then treated as a node in a tree structure, which can be traversed either forwards, allowing one to identify a halo at some early time and follow it forward through the merging hierarchy, or backwards, allowing one to identify a halo and all its progenitors at earlier times. In our analysis, we concentrate on the main trunk of the merger tree, in which we follow the evolution of the main progenitor of a halo to earlier times.

\subsection{Selecting the halo sample}

However, care must be taken when including haloes with masses below $M_{\text {cut }}$ in any analysis. An unfortunate feature of simulations of cosmologies in which small-scale power is suppressed at early times is the formation of unphysical lowmass haloes by the fragmentation of filaments, driven by the discreteness of the matter distribution. These spurious haloes form preferentially in filaments, at regular intervals of order the mean interparticle separation of the simulation, akin to 'beads on a string' (see below). The mass scale below which these spurious 'haloes' form can be estimated from the halo mass function as a sharp upturn in the number density, and it has been shown to scale as $M_{\mathrm{lim}} \sim 3.9 \mathrm{~m}^{1 / 3}{ }_{p} M_{\text {cut }}{ }^{2 / 3}$, where $m_{p}$ is the particle mass (Wang \& White 2007).

We wish to identify haloes in the truncated models that have clearly identifiable counterparts in the fiducial $\Lambda \mathrm{CDM}$ simulation. These haloes form the halo sample upon which our analysis is based. By selecting haloes in this way, we can track the merger trees of the counterparts and study the merging and accretion histories of individual systems, correlating any differences in halo properties with the details of their mass assembly. We can also avoid including in our analysis spurious (unphysical) haloes that form below the mass cut-off in the truncated models (see, for example, Wang \& White 2007).

To identify counterparts, we adapt our algorithm for linking haloes across time slices when building merger trees to link haloes between runs at a given time.

- For each pair of group catalogues, we process each group and compute 'virial' quantities, namely the virial mass and radius, and the set of particles that belong to each halo.

- For each halo in the fiducial $\Lambda \mathrm{CDM}$ model at time $t$, we loop over its associated particles and determine how many of these particles are present in haloes in the corresponding truncated model catalogue. 


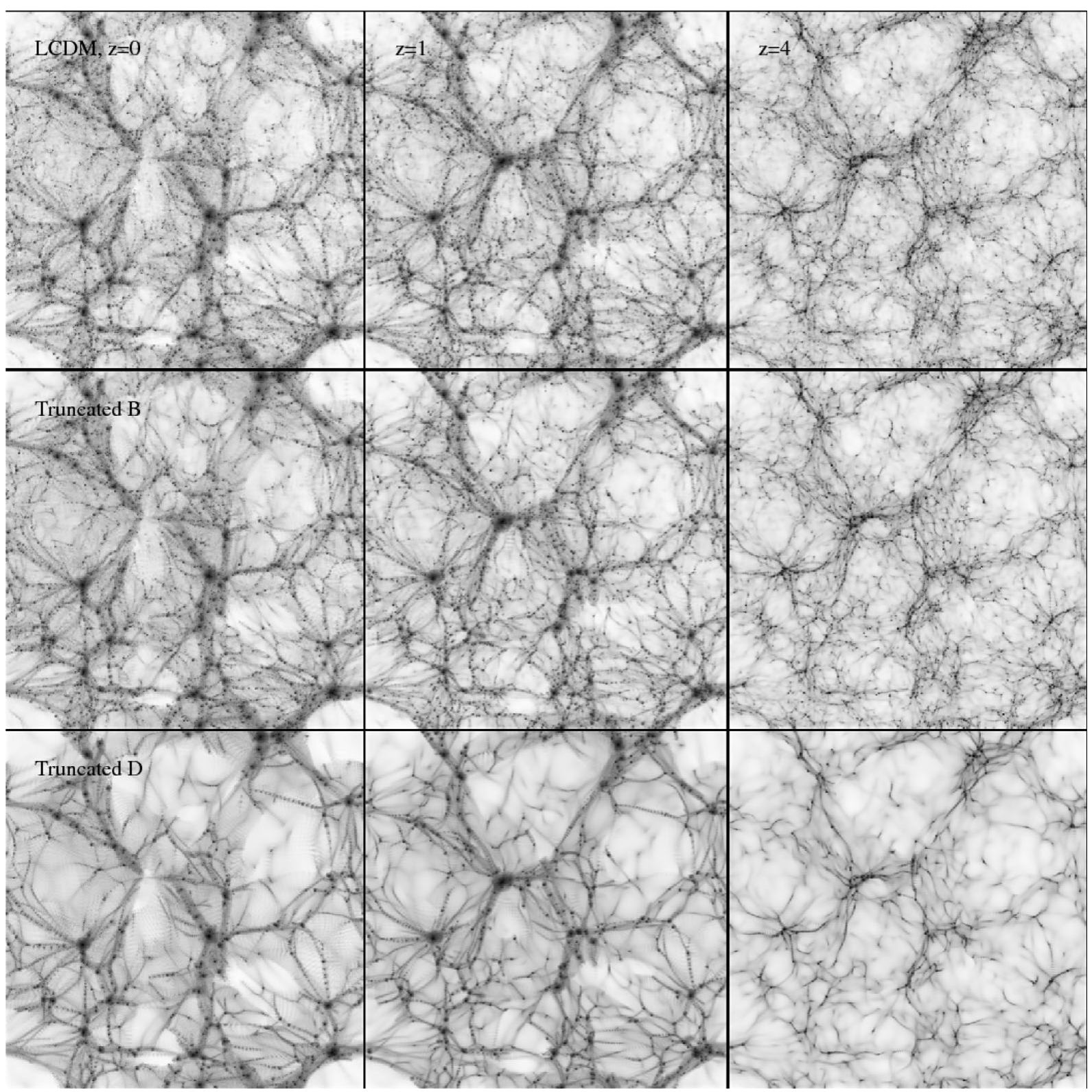

Figure 1. The projected density distribution in $2 h^{-1} \mathrm{Mpc}$ slices taken through the centres of each of the boxes. We have smoothed the particle mass using an adaptive Gaussian kernel and projected onto a mesh. Each mesh point is weighted according to the logarithm of its projected surface density, and so the 'darker' the mesh point, the higher the projected surface density.

- The halo in the truncated model that contains in excess of $f_{\text {count }}=75 \%$ of these particles is identified as a counterpart candidate. However, the candidate halo can be part of a much larger structure in the fiducial $\Lambda \mathrm{CDM}$ model, so we also require that the mass of the candidate halo not differ from its CDM counterpart by not more than a factor of $25 \%$. Haloes that satisfy these conditions are identified as counterparts.

\section{SPATIAL CLUSTERING}

As our starting point, we compare and contrast the spatial clustering of dark matter haloes in the $\Lambda \mathrm{CDM}$ and trun- cated models respectively as a function of redshift. We expect differences between models to be apparent for haloes with masses $M \sim M_{\text {cut }}$ and to become more pronounced with increasing redshift, when $M_{\text {cut }}$ is a larger fraction of the typical collapsing mass $M^{*}$.

\subsection{Visual impression}

In Figure 1, we show the projected dark matter distribution in thin slices $\left(20 \times 20 \times 2 h^{-3} \mathrm{Mpc}^{3}\right)$ taken through the $\Lambda \mathrm{CDM}$ (upper panels), Truncated $\mathrm{B}$ (middle panels; hereafter TruncB), and D (lower panels; hereafter TruncD) at $z=0,1$, and 4 (from left to right). Each slice is 
centred on the geometric centre of the simulation volume and the grey scale is weighted by the logarithm of projected density.

Figure 1 is instructive because it provides a powerful visual impression of the effect of suppressing small-scale power at early times. The filamentary network is largely unaffected and the positions of the most massive haloes, which form at the nodes of these filaments, are similar in each of the models we have looked at. What is striking, however, is the impact on the abundance of low-mass haloes, which appear as small dense knots in projection. As $M_{\text {cut }}$ increases, the projected number density of these low-mass haloes decreases markedly as we go from the $\Lambda \mathrm{CDM}$ run to the TruncD run (top and bottom panels, respectively). This is evident in the clustering around more massive haloes and the absence of low-mass systems in the void regions. Furthermore, the contrast between the models becomes increasingly noticeable with increasing redshift-compare $z=0$ and $z=4$. Note also the presence of the low-mass haloes distributed along filaments in 'beads-on-a-string' fashion in the truncated models.

\subsection{Spatial clustering}

In Figure 2, we investigate how the clustering strength of haloes differs between the different dark matter models and as a function of redshift. We quantify clustering strength by the correlation function $\xi(r)$, which measures the excess probability over random that a pair of haloes $i$ and $j$ will be separated by a distance $r=|\mathcal{E}|=\left|\mathcal{E}_{i}-\mathcal{E}_{j}\right| \cdot \xi(r)$ is estimated using

$$
\xi(r)=1+\frac{\overline{D D(r)}}{\overline{R R(r)}},
$$

where $\overline{D D(r)}$ is the number of haloes at comoving separation

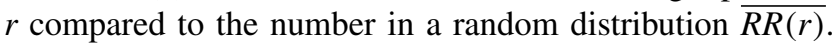
Because our focus is on differences, we construct the ratio of $N(r)=\overline{D D(r)}=\overline{R R(r)}(\xi(r)-1)$ for each truncated model to $N(r)_{\mathrm{CDM}}$ for the fiducial $\Lambda \mathrm{CDM}$ run.

In the upper panel of Figure 2, we consider pairs of haloes in which the primary's mass is $M_{\mathrm{vir}} \geqslant 10^{11} h^{-1} \mathrm{M}_{\odot}$ and the secondary's mass is $M_{\text {vir }} \geqslant 3 \times 10^{9} h^{-1} \mathrm{M}_{\odot}$, while in the lower panel, we consider pairs of haloes in which both the primary and secondary masses $M_{\text {vir }} \geqslant 10^{11} h^{-1} \mathrm{M}_{\odot}$. This reveals that the clustering strength of low-mass haloes around highmass haloes (i.e. $M_{\text {vir }} \geqslant 10^{11} h^{-1} \mathrm{M}_{\odot}$ ) decreases with increasing $M_{\text {cut }}$, although the dependence on $M_{\text {cut }}$ does not appear to be straightforward. In the TruncA and TruncB runs, we find that $N(r) / N(r)_{\mathrm{CDM}}$ is close to unity out to $r \simeq 10 h^{-1} \mathrm{Mpc}$, never deviating by more than $10 \%$ to within $\sim 500 h^{-1} \mathrm{kpc}$ at all redshifts. For the TruncC and TruncD runs, the suppression in clustering strength is quite marked-by $\sim 40 \%$ for the TruncC run and $\sim 50 \%$ for the TruncD run. Large deviations at small radii reflect the small numbers of very close pairs. In contrast, the clustering strength of massive haloes (i.e. $M \geqslant 10^{11} h^{-1} \mathrm{M}_{\odot}$ ) does not appear to be affected by $M_{\text {cut }}$, as
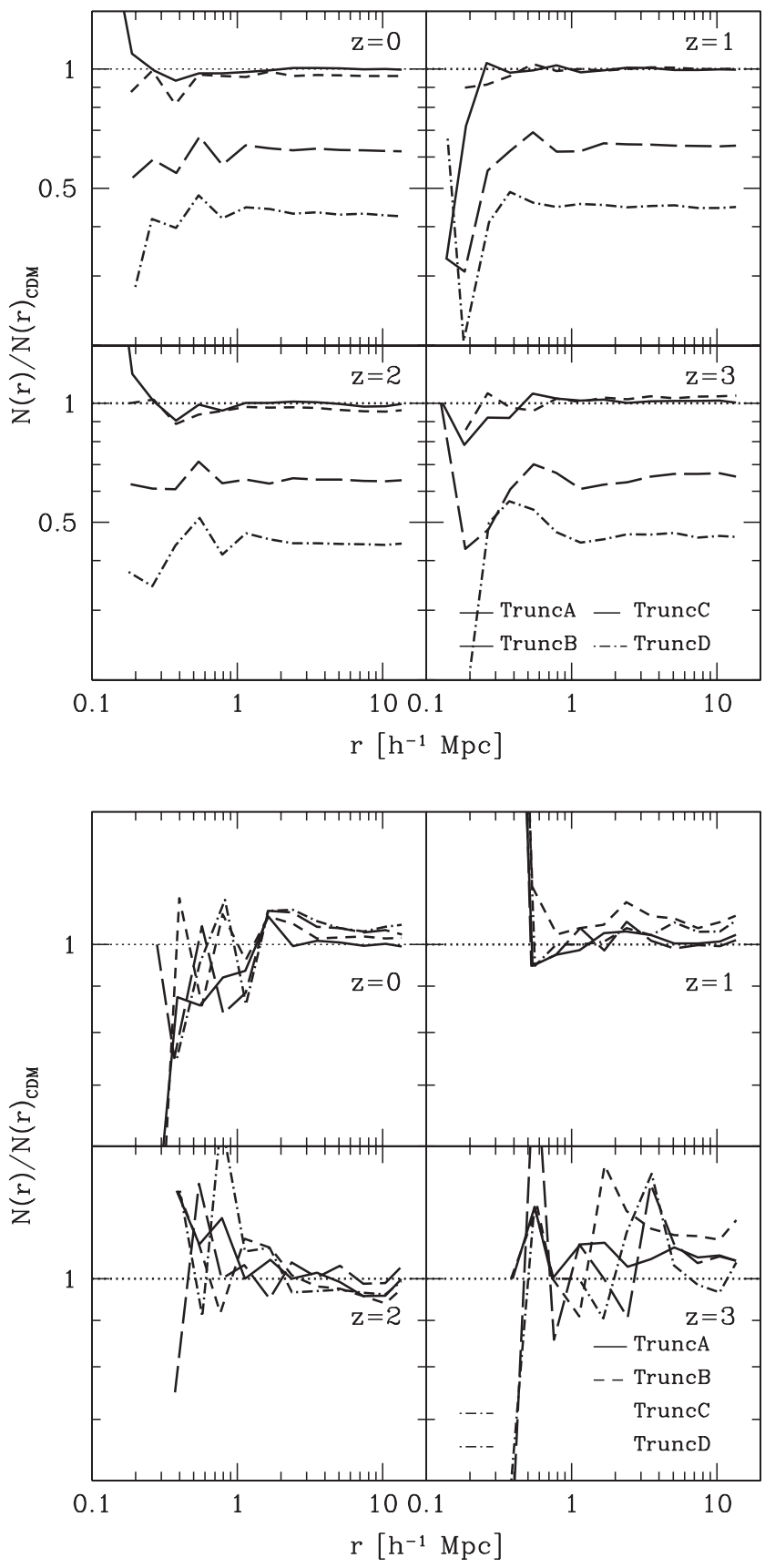

Figure 2. Evolution of spatial clustering with redshift. We examine how the clustering strength of haloes with respect to the fiducial $\Lambda \mathrm{CDM}$ model varies across the runs TruncA (solid curves), TruncB (short-dashed curves), TruncC (long-dashed curves), and TruncD (dotted-dashed curves) at $z=0$, 1,2 , and 3 by plotting the ratio $N(r) / N(r)_{\mathrm{CDM}}$ - the number of haloes with comoving halo separation $r$-as a function of $r$. In the upper panel, we look at the clustering of all secondary haloes with mass $M_{\text {vir }} \geqslant 3 \times 10^{9} h^{-1} \mathrm{M}_{\odot}$ around primary haloes with mass $M_{\text {vir }} \geqslant 10^{11} h^{-1} \mathrm{M}_{\odot}$, while in the lower panel we look at the clustering of only massive haloes, for which both the primary and secondary masses are $M_{\mathrm{vir}} \geqslant 10^{11} h^{-1} \mathrm{M}_{\odot}$.

we inferred from Figure 1. The ratio $N(r) / N(r)_{\mathrm{CDM}}$ is noisyreflecting the lower number density of massive haloes-but it is approximately unity between $0 \lesssim z \lesssim 3$. 


\section{MASS ACCRETION AND MERGING HISTORIES}

Suppressing small-scale power at early times leads to a reduction in the clustering of low-mass haloes around massive haloes $\left(M_{\text {vir }} \gtrsim 10^{11} h^{-1} \mathrm{M}_{\odot}\right)$ at $z \lesssim 3$, which implies that the number of likely minor mergers a typical halo will experience during a given period should decline with increasing $M_{\text {cut }}$. We expect this to depend on both halo mass and epoch. At a given $z$, the merging history of haloes with masses $M_{\text {vir }} \sim M_{\text {cut }}$ should be more sensitive to the clustering of small-scale structure than haloes with masses $M_{\text {vir }} \gg M_{\text {cut }}$. Similarly, at earlier times when the typical collapsing mass $M^{*}$ is smaller and $M_{\text {cut }}$ is a larger fraction of $M^{*}$, we would expect the effect of suppressing small-scale structure to be more pronounced.

When computing mass accretion and merging rates, we use merger trees for all haloes between $5 \times 10^{10} h^{-1} \mathrm{M}_{\odot}(\sim 1$ 600 particles) and $10^{13} h^{-1} \mathrm{M}_{\odot}$ at $z=0$. Note that we have a hard lower limit of 100 particles for a halo to be retained in our catalogues; this corresponds to a mass of $\sim 3.2 \times$ $10^{9} h^{-1} \mathrm{M}_{\odot}$, and so we cannot identify minor mergers with mass ratios of less than $\sim 6 \%$ in our most poorly resolved haloes.

\subsection{Mass accretion rate}

In Figure 3, we show how the mass accretion rate of the most massive progenitors of haloes identified at $z=0$ evolves with redshift. Note that his accretion rate includes both smooth accretion and minor and major mergers. The distinction between minor mergers and smooth accretion may be a moot one in the CDM model - as the mass and force resolution of the simulation increases, we continue to resolve increasing numbers of low-mass haloes-but this is not necessarily the case in the truncated models that we consider.

From upper to lower panels, we show the average accretion rate as a function of redshift for haloes with virial masses at $z=0$ in the range $5 \times 10^{10} \leqslant M_{\text {vir }} / h^{-1} \mathrm{M}_{\odot} \leqslant$ $10^{11}$ (filled circles), $10^{11} \leqslant \mathrm{M}_{\text {vir }} / \mathrm{h}^{-1} \mathrm{M}_{\odot} \leqslant 5 \times 10^{11}$ (filled squares), $5 \times 10^{11} \leqslant M_{\text {vir }} / h^{-1} \mathrm{M}_{\odot} \leqslant 10^{12}$ (filled triangles), and $M_{\text {vir }} / h^{-1} \mathrm{M}_{\odot} \leqslant 10^{12}$ (crosses). Note that we measure the accretion rate as the change in virial mass $(\Delta M)$ per unit redshift $(\Delta z)$ per unit time $(\Delta t)$, normalised by the final (i.e. $z=0$ ) virial mass. Bars indicate r.m.s. scatter.

Figure 3 shows that haloes accrete their mass at similar rates across the different models, regardless of whether or not small-scale power is suppressed at early times. On average, less massive haloes tend to have higher accretion rates at $z \gtrsim 1$ than their more massive counterparts, but this rate starts to drop $z \sim 1$ and declines steadily to $z=0$ (see also Figure 9 for detailed mass accretion histories for individual haloes). In contrast, more massive haloes accrete their mass at a steady rate. We find that our accretion rates for $\triangle \mathrm{CDM}$ haloes are in good agreement with those consistent with those of, for example, Maulbetsch et al. (2007).

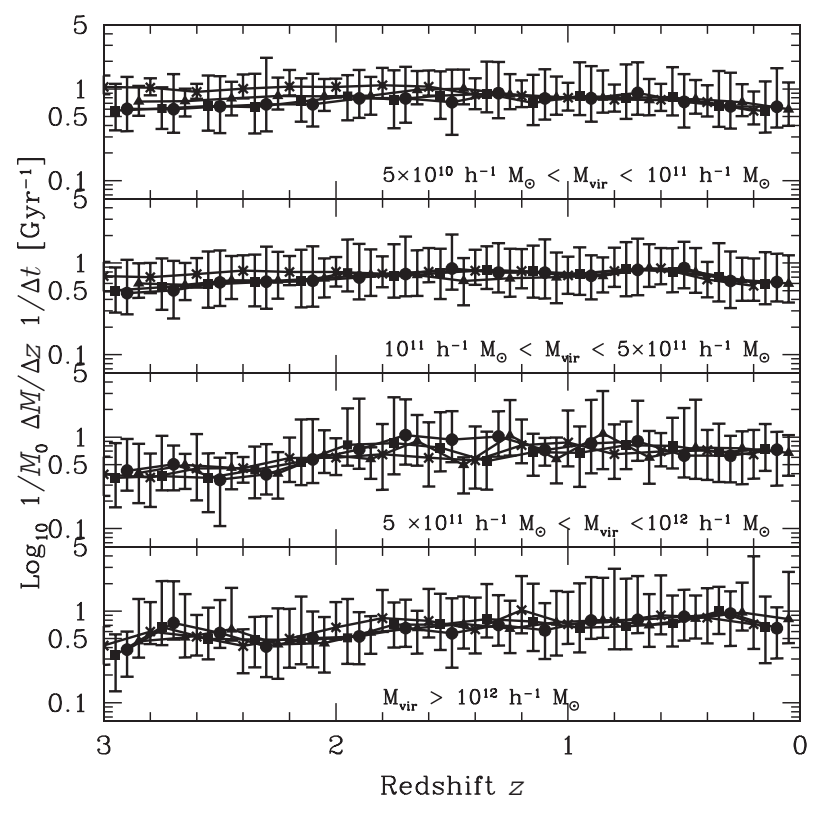

Figure 3. Impact on mass accretion rate. For each halo at $z=0$, we follow the main branch of its merger tree to higher redshifts and compute the difference in virial mass between progenitors at $z_{0}$ and $z_{1}>z_{0}$. From this, we compute the mass accretion rate with respect to time (in Gyrs), normalised by the virial mass of the descendent halo at $z=0$. Within each of the mass bins, we compute the average mass accretion rate for haloes in the fiducial $\lambda \mathrm{CDM}$ run (red-filled circles), TruncB $\left(M_{\text {cut }}=10^{10} h^{-1} \mathrm{M}_{\odot}\right.$; greenfilled squares $)$, TruncC $\left(M_{\text {cut }}=5 \times 10^{10} h^{-1} \mathrm{M}_{\odot}\right.$; cyan-filled triangles $)$, and TruncD $\left(M_{\text {cut }}=10^{11} h^{-1} \mathrm{M}_{\odot} ;\right.$ magenta crosses $)$.

\subsection{Merger rates}

In Figure 4, we focus on the merger rate $\Delta N / \Delta z / \Delta t$ and its variation with redshift, where $\Delta N$ is the number of mergers per unit redshift per unit time. Here, differences between runs are immediately apparent and in the sense that we expect-for halo masses close to $M_{\text {cut }}$ increases, the merging rate decreases. Note that the estimated merger rate is quite noisy in the lowest mass bin (upper panel), especially at early times - in this case, the lower limit of 100 particles imposed by our halo catalogues corresponds to a merger of progressively greater mass ratio with increasing redshift. For this reason, we focus on haloes with masses at $z=0$ in excess of $10^{11} h^{-1} \mathrm{M}_{\odot}$. For haloes with masses between $10^{11} \leqslant M_{\text {vir }} / h^{-1} \mathrm{M}_{\odot} \leqslant 5 \times 10^{11}$, we find that the average merger rate in the TruncC (TruncD) model is a factor of $\sim 3(1.5)$ smaller than that in the fiducial $\Lambda$ CDM model, and this is approximately constant with redshift. The difference is less pronounced for haloes with masses between $5 \times 10^{11} \leqslant M_{\text {vir }} / h^{-1} \mathrm{M}_{\odot} \leqslant 10^{12}$, and for haloes with masses in excess of $10^{12} h^{-1} M_{\odot}$ there is no discernible difference in the merging rates with redshift.

In Figure 5, we assess how major mergers are affected by suppression of small-scale power at early times. This demonstrates that the likelihood that the mass ratio of the most significant merger experienced by a halo since $z \simeq 0.5$ does not depend strongly on whether or not small-scale structure has 


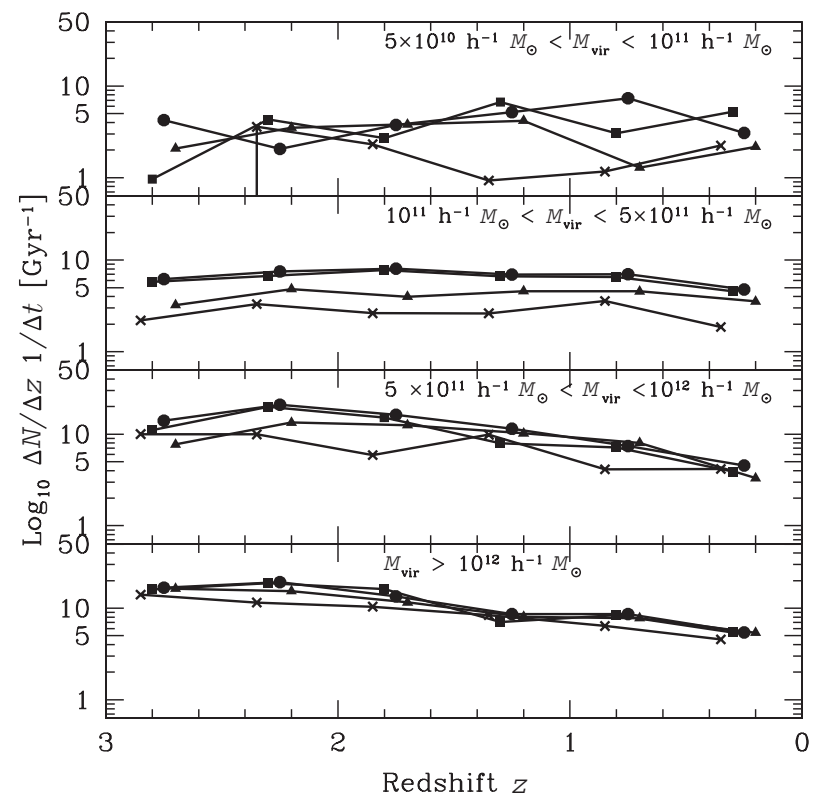

Figure 4. Impact on merger rate. For each halo at $z=0$, we follow the main branch of its merger tree to higher redshifts and determine the number of mergers with mass ratios in excess of $6 \%$ experienced by the halo between $z_{0}$ and $z_{1}>z_{0}$. From this, we compute the merger rate per unit redshift per unit time. Within each of the mass bins, we compute the average merger rate for haloes in the fiducial $\lambda \mathrm{CDM}$ run (red-filled circles), TruncB $\left(M_{\text {cut }}=\right.$ $10^{10} h^{-1} \mathrm{M}_{\odot}$; green-filled squares), TruncC $\left(M_{\text {cut }}=5 \times 10^{10} h^{-1} \mathrm{M}_{\odot}\right.$; cyan-filled triangles $)$, and TruncD $\left(M_{\text {cut }}=10^{11} h^{-1} \mathrm{M}_{\odot} ;\right.$ magenta crosses).

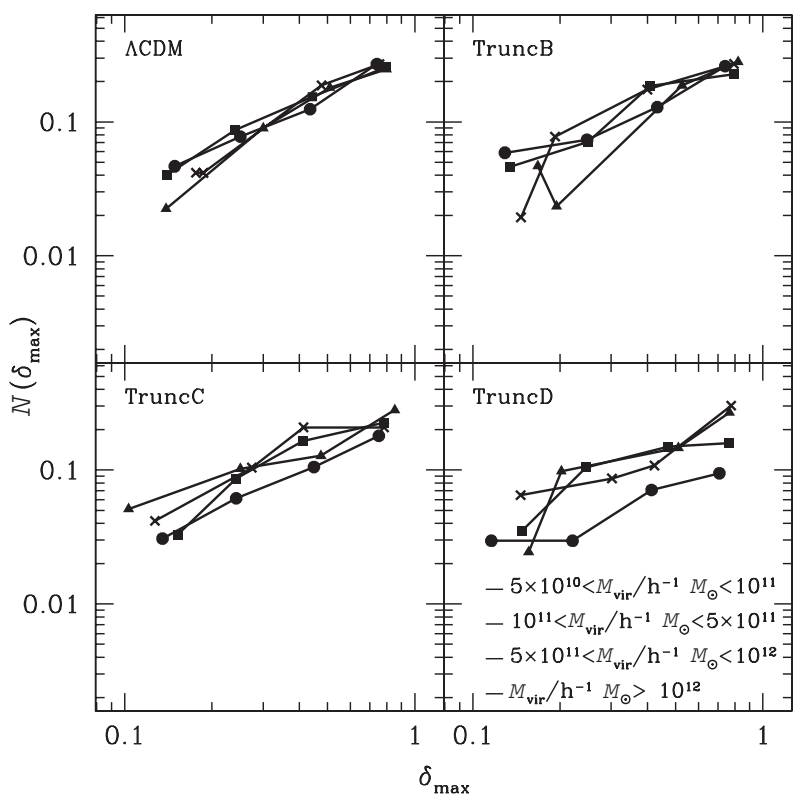

Figure 5. Distribution of most significant mergers. For each halo at $z=0$, we compute the mass ratio of the most significant merger $\delta_{\max }$ that it has experienced since $z \simeq 0.5$ and construct the frequency distribution of $\delta_{\max }$ for the respective models. been suppressed. Here, we follow Power et al. (2012) and compute the distribution of the most significant merger $\delta_{\max }$ $=M_{\text {acc }} / M_{\text {vir }}$ experienced by each halo (identified at $z=0$ ) since $\sim 0.5^{2}$, split according to virial mass at $z=0$.

There are a number of interesting trends in this figure. The first is that most significant mergers with large mass ratios (i.e. minor mergers) are relatively uncommon; the probability distribution increases approximately as a power law with $\delta_{\max }$ as $\delta^{1.2}$ max . The second is that, in the CDM model, the likelihood that a halo experiences a most significant merger with a given $\delta_{\max }$ does not depend strongly on its mass. For example, a halo with virial mass of $10^{11} h^{-1} \mathbf{M}_{\odot}$ is as likely to have experienced a major merger with mass ratio of $\sim 50 \%$ as a $10^{13} h^{-1} M_{\odot}$ halo-approximately $20 \%$. The third is that there is some evidence that haloes in the mass range $5 \times 10^{10} \leqslant M_{\text {vir }} / h^{-1} \mathrm{M}_{\odot} \leqslant 10^{11}$ are less likely to experience major mergers with mass ratios in excess of $\sim 50 \%$ (compare TruncB and TruncD).

\section{ANGULAR MOMENTUM CONTENT}

Suppressing small-scale power at early times impacts both the clustering strength of low-mass haloes and the rate of mergers and accretions at later times. Do we see a corresponding influence on the angular momentum content of haloes at later times?

\subsection{Spin parameter}

We begin by considering the spin parameter $\lambda$, which quantifies the degree to which the halo is supported by rotation and which we define using the 'classical' definition of Peebles (1969),

$$
\lambda=\frac{J|E|^{1 / 2}}{G M_{\mathrm{vir}}^{5 / 2}} .
$$

Here $J$ and $E$ are the total angular momentum and binding energy respectively of material with $r_{\text {vir }}$ and $G$ is the gravitational constant. We impose a lower limit of 600 particles within $r_{\text {vir }}\left(M_{\text {vir }} \geqslant 2 \times 10^{10} h^{-1} \mathrm{M}_{\odot}\right)$ when measuring $\lambda$; this ensures that both $J$ and $E$ are unaffected by discreteness effects (cf. Power et al. 2012).

In Figure 6, we show how the median spin of the halo population evolves with redshift. In the upper panel, we focus on the haloes with $M_{\text {vir }} \geqslant 2 \times 10^{10} h^{-1} \mathrm{M}_{\odot}$, while in the lower panel we consider haloes with $M_{\mathrm{vir}} \geqslant 10^{11} h^{-1} \mathrm{M}_{\odot}$. Filled circles, squares, triangles, and crosses represent the median spin of the halo populations in the $\Lambda \mathrm{CDM}$, TruncB, TruncC, and TruncD runs, and error bars indicate the 25th and 75th percentiles of the distribution. This figure suggests that the behaviour of the distribution of $\lambda$ is sensitive to $M_{\text {cut }}$ - systematic differences are apparent in the TruncC and TruncD runs when we include all haloes with

\footnotetext{
${ }^{2}$ This redshift interval corresponds to $\sim 2$ dynamical times; see Power et al. (2012).
} 

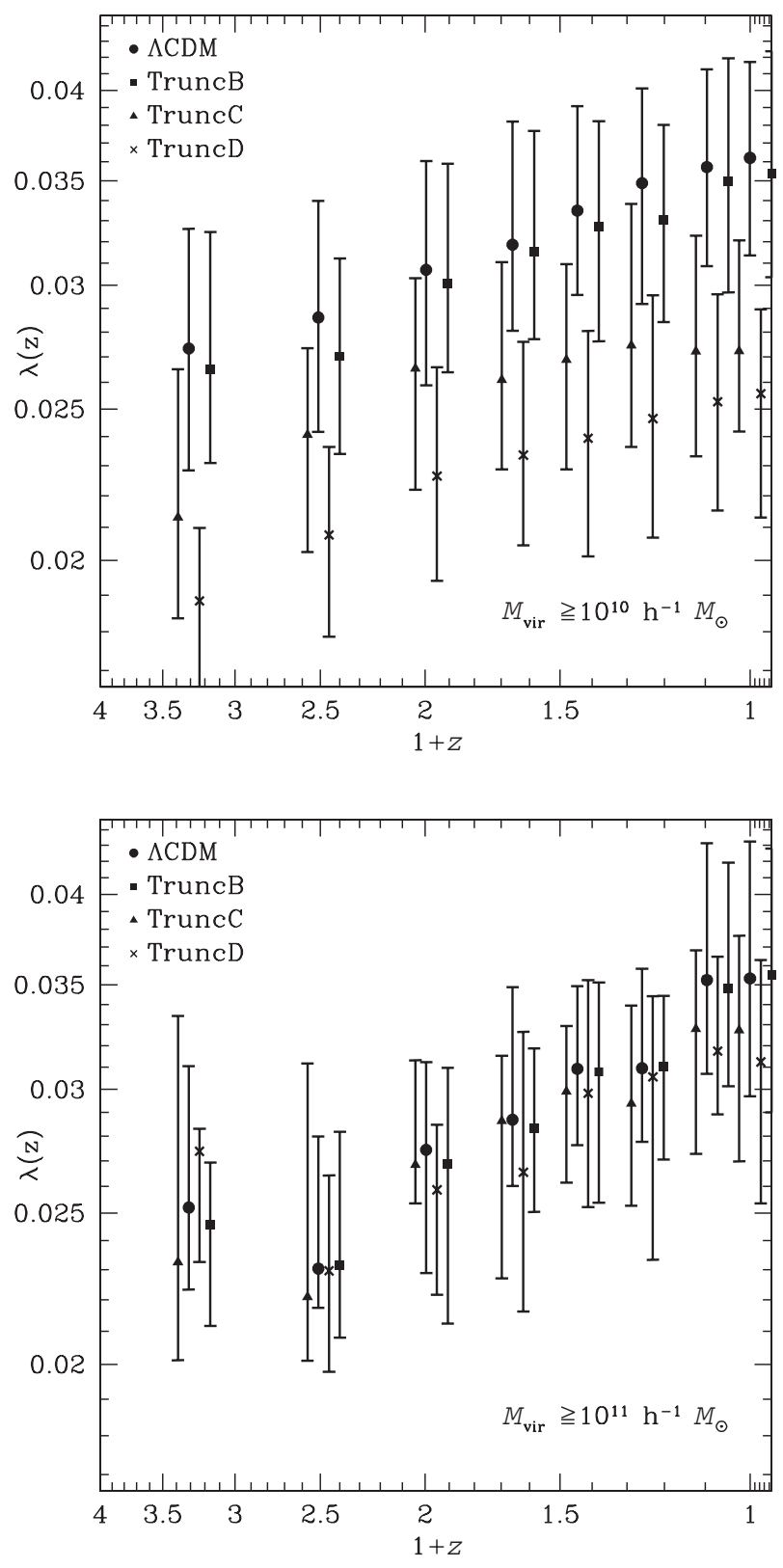

Figure 6. Variation of median $\lambda$ with redshift. We show how the median spin parameter $\lambda_{\text {med }}$ varies with redshift. In the left-hand panel, we consider all haloes with virial masses in excess of $M_{\text {vir }} \geqslant 1.9 \times 10^{10} h^{-1} \mathrm{M}_{\odot}$, while in the right-hand panel, we consider all haloes that satisfy $M_{\text {vir }} \geqslant 10^{11} h^{-1} \mathrm{M}_{\odot}$. Lower and upper error bars represent the 25 th and 75 th percentiles. The filled circles, squares, triangles, and crosses correspond to the fiducial $\Lambda \mathrm{CDM}$, TruncB, TruncC, and TruncD runs, respectively.

$M_{\mathrm{vir}} \geqslant 2 \times 10^{10} h^{-1} \mathrm{M}_{\odot}$, whereas the distributions are statistically similar when we include only haloes with $M_{\text {vir }} \geqslant$ $10^{11} h^{-1} \mathbf{M}_{\odot}$.

This figure is interesting because we include a large population of haloes in the TruncC and TruncD runs with $M_{\text {vir }} \leq M_{\text {cut }}$ when we include haloes with $M_{\text {vir }} \geqslant 2 \times$ $10^{10} h^{-1} \mathrm{M}_{\odot}$, and so the apparent differences are to be expected. In contrast, we do not see any evident differences when we include haloes with $M_{\text {vir }} \geqslant 10^{11} h^{-1} \mathrm{M}_{\odot}$. This is also interesting because it reveals that the median $\lambda$ increases with decreasing redshift at approximately the same rate-in proportion to $(1+z)^{-0.3}$-regardless of whether or not we include haloes with masses below $M_{\text {cut }}$.

In Figure 7, we focus on individual haloes, showing how $\lambda$ and the specific angular momentum $j=J / M$ vary with redshift $z$ for a selection of haloes with quiescent and violent merging histories, drawn from haloes with $M_{\text {vir }} \geqslant 10^{11} h^{-1} \mathrm{M}_{\odot}$ over the redshift interval $0 \leq z \leq 3$. For each halo, we determine the most significant merger $\delta_{\max }$ that it has experienced since $z=1$, where we define $\delta_{\max }$ as the mass ratio of the most major merger experienced by the main progenitor of a halo identified at $z=0$ during the redshift interval $0 \leq z \leq 1$ (cf. Power et al. 2012). This gives a distribution of $\delta_{\max }$ and we identify haloes in the upper (lower) 20\% of the distribution as systems with violent (quiescent) merging histories. For ease of comparison, we focus on the extremes-the $\Lambda \mathrm{CDM}$ and TruncD runs (top and bottom, respectively).

There are a few points worthy of note in relation to the evolution of the spin parameter with redshift. First, the spin parameter for a given halo is a very noisy quantity but if we consider the average behaviour of haloes in the respective samples, we do not find any clear correlation between spin and redshift (based on their Spearman rank coefficient). Second, there is a clear offset between median spins in the quiescent and violent samples-haloes with violent merging histories tend to have higher spins (by factors of $~ 3-4$ ) than haloes with quiescent histories. However, there is appreciable scatter over any given halo's history-the r.m.s. variation is $\sim 0.25-0.29$ for haloes in the quiescent sample and $\sim 0.39-0.42$ in the violent sample. Importantly, third, it is the dynamical state and merging history of a halo that has greater impact on its instantaneous spin and specific angular momentum - the influence of the dark matter is a secondary effect at best.

Note that we also compare the growth of angular momentum and spin for three sets of cross-matched haloes across dark matter models-shown in Figures 8 and 9. From our cross-matched catalogues, we identified blindly a set of three haloes with $M_{\text {vir }} \simeq(7.85,0.61,0.076) \times 10^{12} h^{-1} \mathrm{M}_{\odot}$, which are approximately 1,10 , and 100 times the threshold mass of $M_{\text {cut }}=10^{11} h^{-1} \mathrm{M}_{\odot}$.

Projections of the density distribution in cubes approximately $2 r_{\text {vir }}$ on a side and centred on the haloes are shown in Figure 8-high-, intermediate-, and low-mass haloes (left, middle, and right panels) in the $\Lambda \mathrm{CDM}$, TruncB, TruncC, and TruncD models (top to bottom panels, respectively). Qualitatively, the haloes appear similar, with the decreasing abundance of substructure with increasing severity of truncation in initial $P(k)$ being the key difference between the models. There are small differences in the orientation of the intermediate- and low-mass haloes (compare, for example, the intermediate-mass halo in the TruncC and TruncD runs) and in the positions of subhaloes (compare, for example, the low-mass halo in the TruncB and TruncD runs), but such 

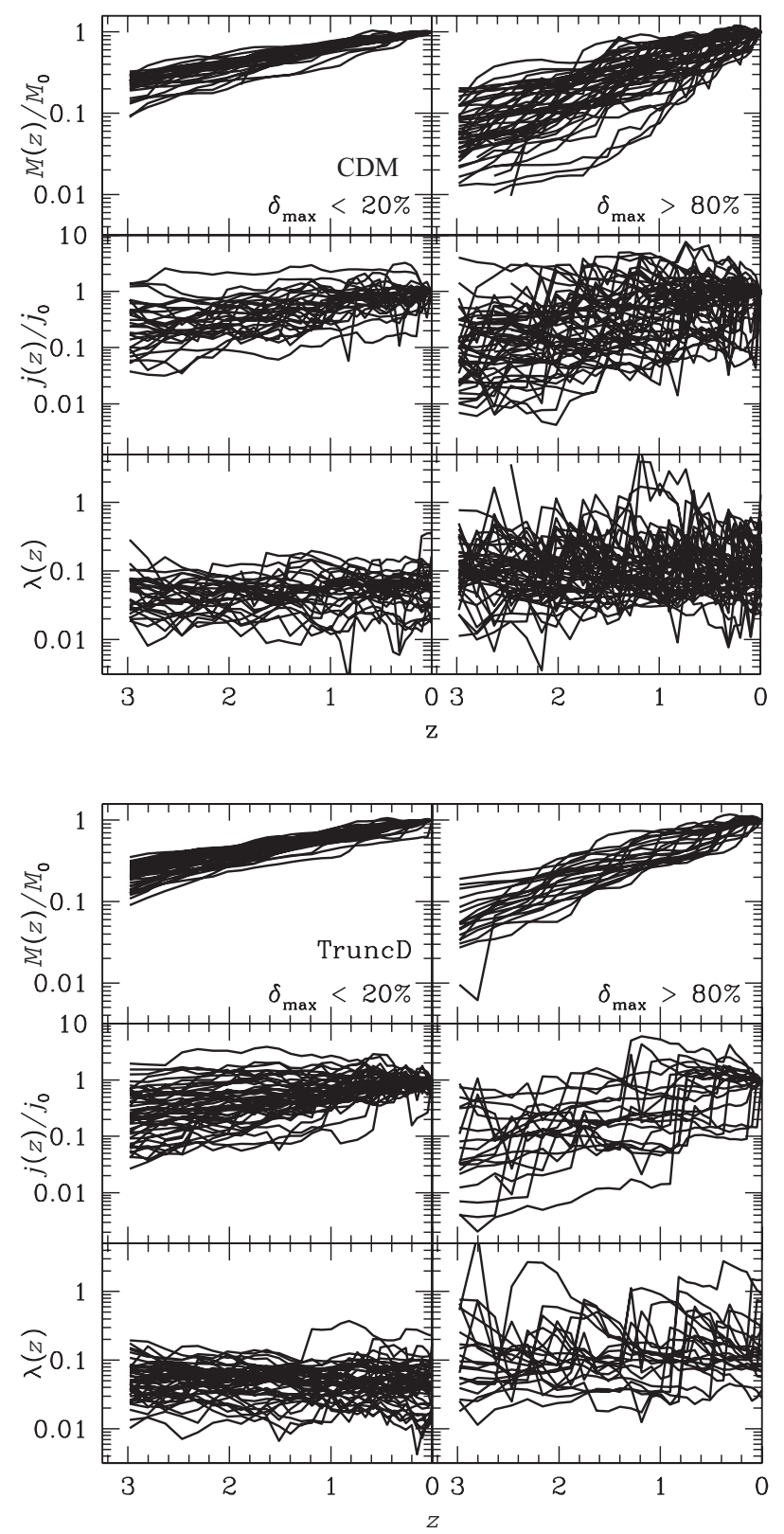

Figure 7. Variation of $\lambda$ and $j$ with redshift for relaxed and unrelaxed haloes. We use the merging histories of haloes to identify two samples of haloes, one with a quiescent merging history ( $\delta_{\max } \lesssim 0.2$ since $z=3.0$; left-hand panels) and one with a violent merging history $\left(\delta_{\max } \geq 0.8\right.$ over the same period; right-hand panels) in the $\Lambda \mathrm{CDM}$ and TruncD runs (upper and lower panels, respectively). Haloes are chosen such that their virial mass at $z=$ 0 satisfies $M_{\text {vir }} \geqslant 10^{11} h^{-1} \mathrm{M}_{\odot}(\sim 3000$ particles $)$. The upper, middle, and lower panels show the growth of halo virial mass (normalised to the virial mass at $z=0$ ), the specific angular momentum $j=J / M$ (normalised to its value at $z=0, j_{0}$ ), and dimensionless spin parameter $\lambda=J|E|^{1 / 2} / G M^{5 / 2}$ vir as a function of redshift $z$.

differences are to be expected at the mass and force resolution of our simulations.

Figure 9 shows in detail how the virial mass (upper panels), specific angular momentum (middle panels), and spin parameter (lower panels) grow over time for each of the three sets of haloes. For the most massive halo, the mass assembly histories are indistinguishable, while the specific angular momentum and spin growth are in very good agreement with each other. For the intermediate-mass halo, there are differences in the mass assembly histories at $z \gtrsim 1$, with the TruncC and Trunc deviating from the $\Lambda \mathrm{CDM}$ and TruncB cases, but they are negligible; the specific angular momentum and spin growth show small differences but they are in good broad agreement. For the low-mass halo, it is noticeable that the mass growth is in good general agreement across the models at $z \lesssim 3$, but the mass of the halo in the TruncD case has to grow rapidly to catch up with its counterparts in the $\Lambda \mathrm{CDM}$, TruncB, and TruncC runs at $z \gtrsim 3$. This has a knock-on effect in the growth of its specific angular momentum and spin parameter; however, the mass, specific angular momentum, and spin parameter growth are in very good agreement for $z \lesssim 1$.

\subsection{Specific angular momentum profiles}

There does not appear to be any systematic difference in the bulk angular momenta of haloes, i.e. the total angular momentum of material within $r_{\mathrm{vir}}$. What of the distribution of angular momentum within $r_{\mathrm{vir}}$ ? We focus on the specific angular momentum profile, which quantifies the fraction of material within the virial radius that has specific angular momentum of $j$ or less. Figure 10 shows the average specific angular momentum profile $M(<j)$ of haloes in each of our models.

We compute specific angular momentum profiles using the method presented in Bullock et al. (2001, 2002). In brief, we compute the total angular momentum of the halo and define this as the $z$-axis; then we sort particles into spherical shells of equal mass and increasing radius, and we assign shell particles to one of three equal volume segments determined by the particle's angle with respect to the $z$-axis; finally, we compute both the total and $z$-component of the specific angular momentum in each segment. This allows us to compute the fraction of halo mass with specific angular momentum of $j$ (and its $z$ component $j_{z}$ ) or less. Note that we scale our profiles by $j_{\max }$, the maximum specific angular momentum that we measure in our data; this is distinct from the $j_{\max }$ used in Bullock et al. $(2001,2002)$, who estimate $j_{\max }$ by fitting their universal angular momentum profile.

In Figure 10, we show the specific angular momentum profile for the total angular momentum $j$, although the $j_{z}$ behaviour is similar. For ease of comparison, we have applied small offsets to the data points from the truncated models. There are a few points worthy of note in this figure. The profile gently curves towards shallower logarithmic slopes with increasing $j$; we find that $M(<j) \sim j^{5 / 2}$ for the lowest angular momentum material and $M(<j) \sim j^{1 / 2}$ for the highest angular momentum material. It is interesting that there is a systematic trend for lower angular momentum material in the $\Lambda \mathrm{CDM}$ and TruncB runs to have on average lower values of $j$ than the TruncC and TruncD runs-the difference 

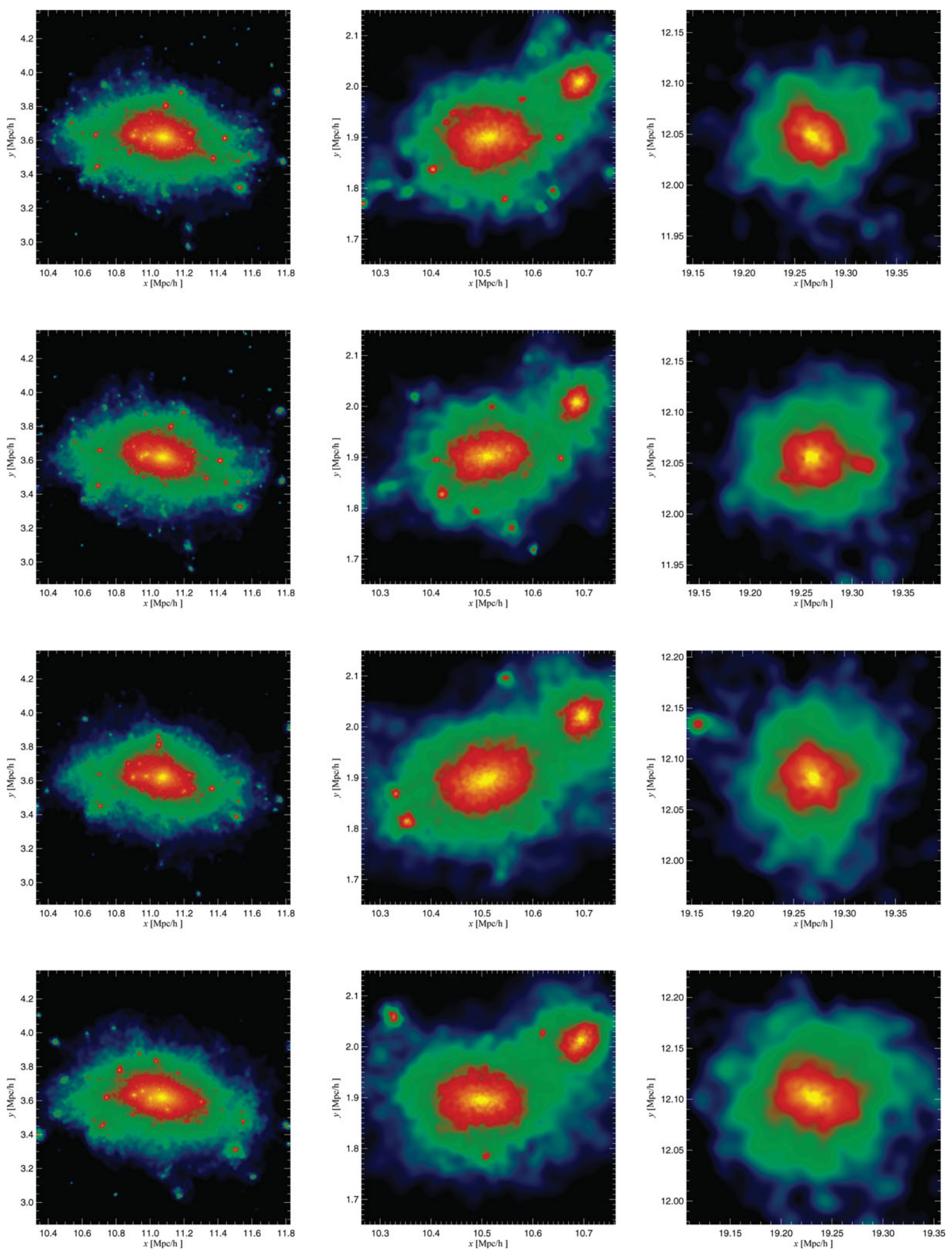

Figure 8. Direct comparison of haloes: projected dark matter density maps. From left to right, haloes with virial masses at $z=0$ of $M_{\text {vir }} \simeq(7.85,0.61,0.076) \times 10^{12} h^{-1} \mathrm{M}_{\odot}$ in the CDM, TruncB, TruncC, and TruncD (from top to bottom).

is of order $25 \%$ at most. This trend is not evident when one looks at the projected specific angular momen$\operatorname{tum}\left(j_{z}\right)$ profile. However, the r.m.s. scatter is large for a given
$M(<j)$ or $M\left(<j_{z}\right)$ in all our models, and for interesting values of $M_{\text {cut }} \sim 10^{9} h^{-1} \mathrm{M}_{\odot}$ (comparable to our TruncA run) there is no statistically significant difference. 

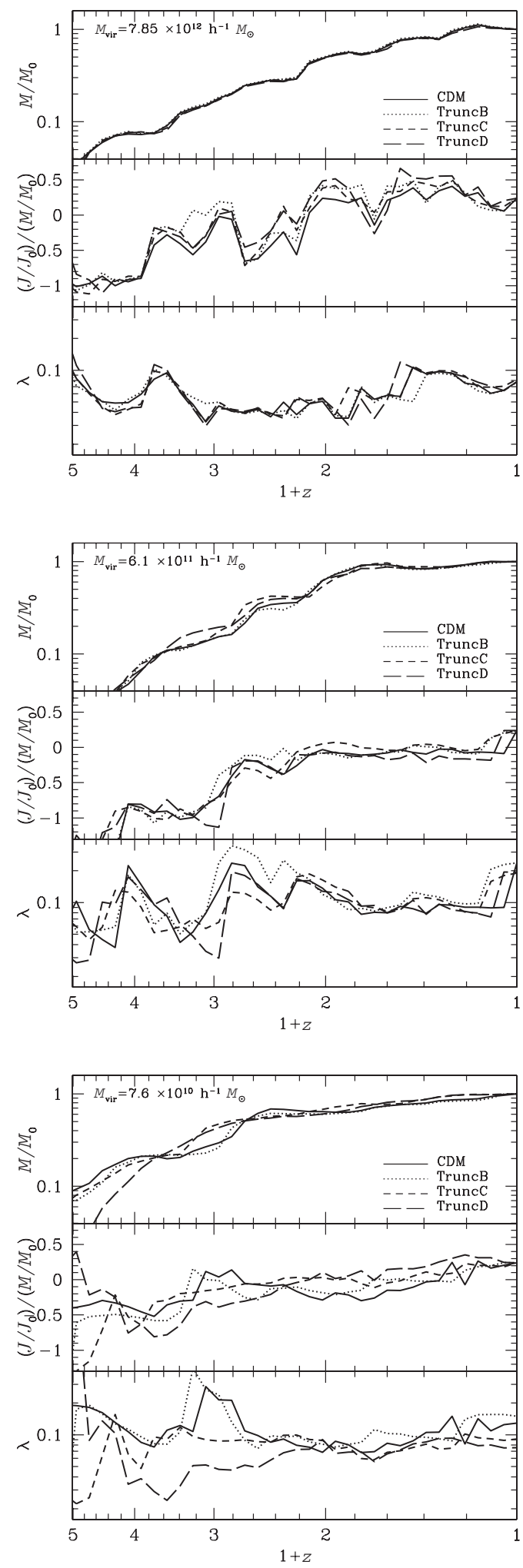

Figure 9. Direct comparison of haloes: redshift evolution of spin and specific angular momentum evolution. Upper/middle/lower panel show growth of virial mass (normalised to $M_{\mathrm{vir}}$ at $z=0$ ), specific angular momentum (normalised to value at $z=0$ ), and spin parameter $\lambda$ as function of $1+z$.

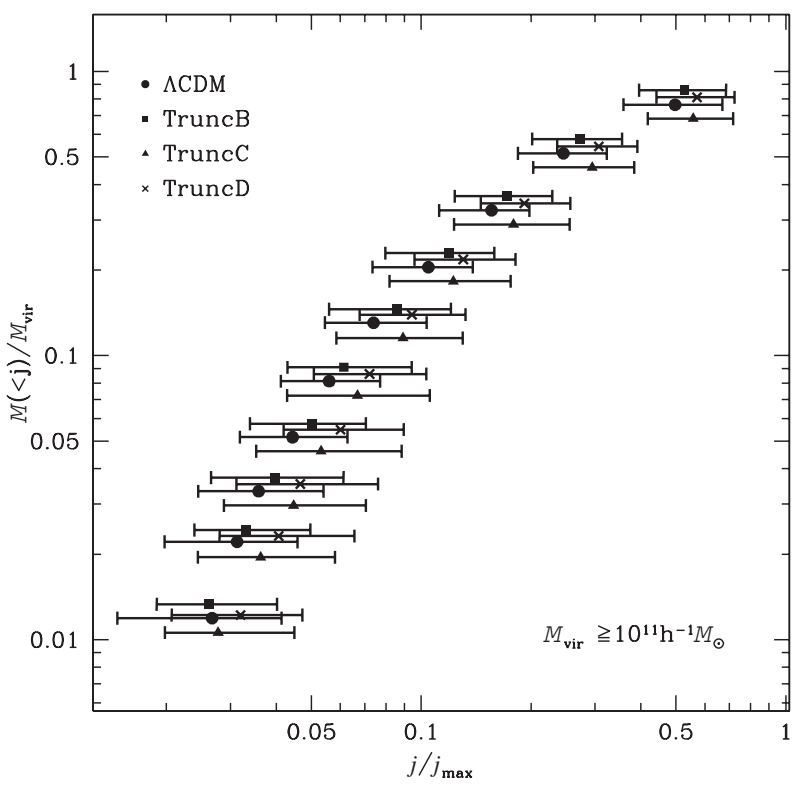

Figure 10. Specific angular momentum profiles. We use the method of Bullock et al. $(2001,2002)$ to determine the fraction of halo mass that has a total specific angular momentum of $j$ or less. Note that we consider only haloes that satisfy $M_{\mathrm{vir}} \geqslant 10^{11} h^{-1} \mathrm{M}_{\odot}$.

\subsection{Angular momentum of the Lagrangian volume}

In Figure 11, we investigate the angular momentum of the Lagrangian region corresponding to the virialised halo at $z=0$ and determine how it evolves with time for haloes with masses in excess of $5 \times 10^{10} h^{-1} \mathrm{M}_{\odot}$ at $z=0$. In other words, we track the angular momentum of all the material that contributes to the final halo at $z=0$. We identify particles at $z=9$ that reside within the virial radius at $z=0$ and compute their angular momentum $\mathcal{E}$ using their centre of mass and centre of mass velocity. In addition, we estimate the mean radial velocity of this material with respect to the centre of mass velocity and determine the redshift at which it changes sign from positive to negative (i.e. from expansion to contraction); this defines the redshift of turnaround $z_{t}$. This is typically between $0.6 \lesssim z \lesssim 4$ for the haloes we consider. This is equivalent to one of the two empirical measures of turnaround employed by Sugerman et al. (2000).

We expect tidal torques arising from gravitational interaction with the surrounding matter distribution to drive the growth of angular momentum at early times (prior to turnaround) and so it should not be particularly sensitive to a small-scale cut-off in the power spectrum. Linear perturbation theory should hold, and the angular momentum of the material should grow in proportion to $(1+z)^{-3 / 2}$ (cf. White 1984). Therefore, we expect the angular momentum at turnaround to be close to its maximum value ${ }^{3}$ and the frequency distribution of angular momenta should be similar

\footnotetext{
${ }^{3}$ Sugerman et al. (2000) have shown that the angular momentum continues to grow 'quasi-linearly' after turnaround until first shell crossing, at which point it reaches its maximum value.
} 

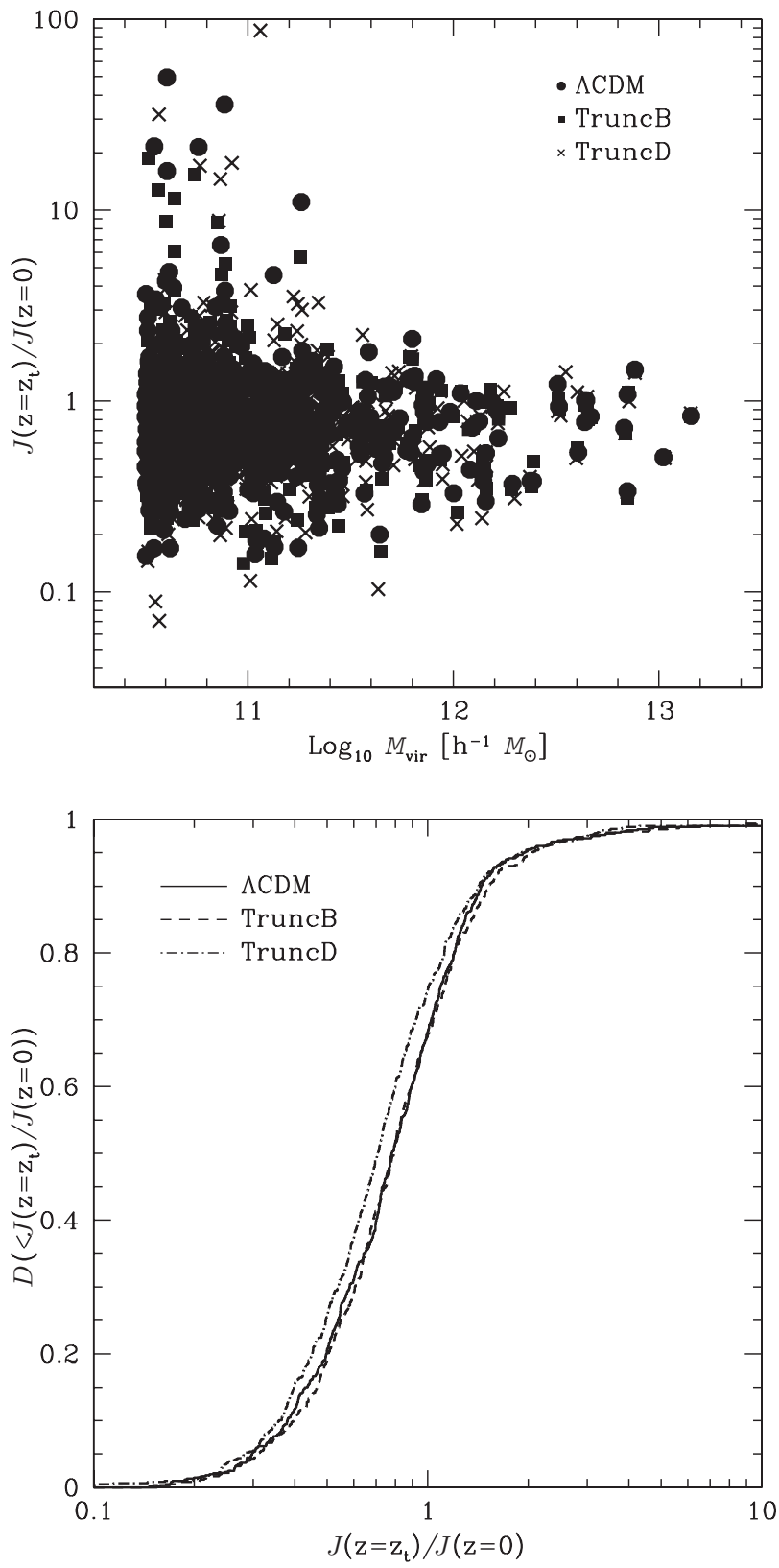

Figure 11. Angular momentum at turnaround. We track the material associated with each halo identified at $z=0$ and compute the radial extent and angular momentum of this material as a function of redshift in the $\Lambda \mathrm{CDM}$, TruncB, and TruncD runs. When the material has reached its maximum radial extent, we denote the epoch at which this occurs as turnaround and look at the ratio of the magnitude of angular momentum of the material at this redshift $z_{\mathrm{t}}, J\left(z_{\mathrm{t}}\right)$, with respect to the magnitude of the angular momentum of this material at $z=0$. In the upper panel, we show the variation of this ratio with halo mass at $z=0$; in the lower panel, we show the cumulative distribution $D\left(<J\left(z_{\mathrm{t}}\right) / J(z=0)\right)$.

in each of the models we have looked at. Linear perturbation theory no longer provides a good description of angular momentum growth subsequent to turnaround and non-linear processes (i.e. mergers) are believed to become more important drivers of angular momentum evolution during this phase. Therefore, if there are differences between the mod- els, we would expect them to be apparent in the ratio of the 'peak' angular momentum at turnaround to the final angular momentum at $z=0$.

In the upper panel of Figure 11, we show the distribution of $J\left(z_{\mathrm{t}}\right) / J(z=0)$ versus halo mass, while in the lower panel we show the cumulative distribution $D\left(<J\left(z_{\mathrm{t}}\right) / J(z=0)\right)$ for all haloes with masses in excess of $5 \times 10^{10} h^{-1} \mathrm{M}_{\odot}$ at $z=0$. For clarity, we consider only the $\lambda \mathrm{CDM}$ (filled circles, solid curves), TruncB (filled squares, dashed curves), and TruncD (crosses, dotted-dashed curves) runs. The upper panel reveals that, on average, the ratio of $J\left(z_{\mathrm{t}}\right) / J(z=0)$ does not vary appreciably with mass and that it is slightly less than unity (approximately 0.8). In other words, the magnitude of the total angular momentum of the material at turnaround is on average smaller than at $z=0$.

These figures reveal that the small differences that we observe in the spin distributions are also present in the specific angular momentum. The median $J\left(z_{\mathrm{t}}\right) / J(z=0)$ differs by $\sim 10 \%$ between the $\Lambda$ CDM model and the TruncD run.

\section{SUMMARY AND DISCUSSION}

The focus of this paper has been to determine the extent to which suppressing the formation of small-scale structurelow-mass dark matter haloes-affects observationally accessible properties of galaxy-mass dark matter haloes. Using cosmological $\mathrm{N}$-body simulations, we have investigated the spatial clustering of low-mass haloes around galaxy-mass haloes, the rate at which these haloes assemble their mass and at which they experience mergers, and their angular momentum content in a fiducial $\Lambda \mathrm{CDM}$ model and in truncated ( $\Lambda$ WDM-like) models. The main results of our study can be summarised as follows:

Large-scale structure: Visual inspection of the density distribution reveals that the structure that forms in truncated models is indistinguishable from that in the $\Lambda \mathrm{CDM}$ model on large scales but differs on small scales. Precisely how small this scale is depends on $M_{\text {cut }}$, the mass scale below which lowmass halo formation is suppressed, which we varied between $5 \times 10^{9} h^{-1} \mathrm{M}_{\odot}$ and $10^{11} h^{-1} \mathrm{M}_{\odot}$. For $M_{\text {cut }}=5 \times 10^{9} h^{-1} \mathrm{M}_{\odot}$, the differences with respect to the $\Lambda$ CDM model are negligible, but they become significant for $M_{\text {cut }}=10^{11} h^{-1} \mathrm{M}_{\odot}$.

Spatial clustering: These visual differences are apparent in the clustering strength of lower-mass secondary haloes around galaxy-mass primaries. Fixing the primary mass at $M_{\mathrm{vir}}=10^{11} h^{-1} \mathrm{M}_{\odot}$, we found that the clustering strength of secondaries around primaries depends strongly on $M_{\text {cut }}$ and the minimum secondary mass. If we include secondaries with masses $M_{\text {vir }} \geq 3 \times 10^{9} h^{-1} \mathrm{M}_{\odot}$, the differences are as great as $\sim 50 \%$ when $M_{\text {cut }}=10^{11} h^{-1} \mathrm{M}_{\odot}$. Unsurprisingly, we found no dependence on $M_{\text {cut }}$ if secondaries are restricted to haloes with masses $M_{\mathrm{vir}} \geq 10^{11} h^{-1} \mathrm{M}_{\odot}$.

Mass accretion and merger rates:. The sensitivity of the clustering strength to $M_{\text {cut }}$ has immediate consequences for 
the frequency of minor mergers. The effect is most striking for models with $M_{\text {cut }} \geq 5 \times 10^{10} h^{-1} \mathrm{M}_{\odot}$, when the rate of all mergers with mass ratios in excess of $\sim 6 \%$ is suppressed across all redshifts by factors of $\sim 2$ to 3 in haloes with virial masses of $M_{\text {vir }} \lesssim 5 \times 10^{11} h^{-1} \mathrm{M}_{\odot}$. This effect must be driven by a reduction in the number of minor mergers because the frequency of major mergers does not depend on $M_{\text {cut }}$ other than in haloes with masses $M_{\mathrm{vir}} \sim M_{\text {cut }}$. Interestingly, we found that the total mass accretion rate does not appear to be sensitive to $M_{\text {cut }}$ at all.

Halo angular momentum: . Minor mergers appear to have little influence on the angular momentum content of galaxymass haloes.

1. We computed the spin parameter $\lambda$ and found no obvious dependence on $M_{\text {cut }}$ but a strong dependence on mass accretion history has-a marked systematic offset is evident between the average spins of haloes with violent mass accretion histories and those with quiescent histories - by a factor of $\sim 2$ to 3 , independent of $M_{\text {cut }}$. The spin of individual haloes evolves in an almost stochastic fashion over time and on average do not show any obvious evolution with redshift.

2. We examined the angular momentum distribution within haloes by constructing specific angular momentum profiles, which quantify the fraction of material within a halo that has specific angular momentum of $j$ or less. We found a weak trend for halo material in truncated models with values of $M_{\text {cut }}$ greater than $10^{10} h^{-1} \mathrm{M}_{\odot}$ to have on average smaller values of $j$ by $25 \%$ at most, but the r.m.s scatter is large for a given $M(<j)$ in all our models and the differences have a low statistical significance.

3. We investigated the angular momentum of the Lagrangian region corresponding to the virialised halo at $z=0$ and determined how it evolves with time. We calculated $J\left(z_{\mathrm{t}}\right) / J(z=0)$, the ratio of the angular momentum of the material at the turnaround redshift $z_{\mathrm{t}}$ to $z=0$. Again the differences between the models are small, at most $10 \%$.

These results indicate that small-scale structure has little impact on the angular momentum content of galaxy-mass haloes, in broad agreement with those of Wang and White (2009), who studied halo formation in hot dark matter models, and Bullock et al. (2002) and Chen and Jing (2002), who looked at WDM models.

These results show that there are differences in the spatial clustering and merger rates of low-mass haloes between our fiducial $\Lambda \mathrm{CDM}$ model and the truncated models-but that they are evident only in the most extreme truncated models, with $M_{\text {cut }}$ in excess of $10^{10} h^{-1} \mathrm{M}_{\odot}$. As we noted in the introduction, this is inconsistent with astrophysical constraints on the putative WDM particle mass. Therefore, measuring the effect on spatial clustering or the merger rate is likely to be observationally difficult for realistic values of $M_{\text {cut }}$, equivalent to our TruncA runs, and so isolating the effect of this small-scale structure would appear to be remarkably difficult to detect, at least in the present day Universe.

However, there are important caveats. The effect may not be so subtle in the high redshift Universe, during the earliest epoch of galaxy formation, and so we might expect marked differences in the abundances of low-mass satellites between our fiducial $\Lambda \mathrm{CDM}$ model and WDM or truncated models. This may have observable consequences for the ages and metallicities of the oldest stars in galaxies (e.g. Frebel 2005), the abundance of metal poor globular clusters, and the assembly of galaxy bulges and stellar haloes. In addition, there is no compelling reason to expect that the efficiency of galaxy formation will differ between a $\Lambda \mathrm{CDM}$ model and a WDM or truncated model, and so it may be the case that galaxy formation in a WDM(-like) model is easier to reconcile with the observed galaxy population than galaxy formation in the fiducial $\Lambda$ CDM model (see also Menci et al. 2012; Benson et al. 2013). We shall return to these ideas in forthcoming work.

\section{ACKNOWLEDGEMENTS}

$\mathrm{CP}$ thanks the anonymous referee for their thoughtful report. This work was supported by ARC DP130100117 and by computational resources on the EPIC supercomputer at iVEC through the National Computational Merit Allocation Scheme. The research presented in this paper was undertaken as part of the Survey Simulation Pipeline (SSimPL; http: //ssimpl-universe.tk/).

\section{REFERENCES}

Angulo, R. E., Hahn, O., \& Abel, T. 2013, MNRAS, 434, 3337

Bailin, J., \& Steinmetz, M. 2005, ApJ, 627, 647

Bardeen, J. M., Bond, J. R., Kaiser, N., \& Szalay, A. S. 1986, ApJ, 304, 15

Benson, A. J., et al. 2013, MNRAS, 428, 1774

Benson, A. J., Lacey, C. G., Baugh, C. M., Cole, S., \& Frenk, C. S. 2002, MNRAS, 333, 156

Bergström, L. 2000, RPPh, 63, 793

Bode, P., Ostriker, J. P., \& Turok, N. 2001, ApJ, 556, 93

Boehm, C., Mathis, H., Devriendt, J., \& Silk, J. 2005, MNRAS, 360,282

Boylan-Kolchin, M., Bullock, J. S., \& Kaplinghat, M. 2012, MNRAS, 422, 1203

Bullock, J. S., Kolatt, T. S., Sigad, Y., Somerville, R. S., Kravtsov, A. V., Klypin, A. A., Primack, J. R., \& Dekel, A. 2001, MNRAS, 321,559

Bullock, J. S., Kravtsov, A. A. V., \& Colín, P. 2002, ApJ, 564, L1

Cantalupo, S. 2010, MNRAS, 403, L16

Chen, D. N., \& Jing, Y. P. 2002, MNRAS, 336, 55

Cole, S., \& Lacey, C. 1996, MNRAS, 281, 716

Colín, P., Avila-Reese, V., Valenzuela, O., \& Firmani, C. 2002, ApJ, 581,777

Colín, P., Valenzuela, O., \& Avila-Reese, V. 2008, ApJ, 673, 203 
Davé, R., Spergel, D. N., Steinhardt, P. J., \& Wandelt, B. D. 2001, ApJ, 547, 574

Dekel, A., \& Silk, J. 1986, ApJ, 303, 39

Dunstan, R. M., Abazajian, K. N., Polisensky, E., \& Ricotti, M. 2011, ArXiv e-prints

Efstathiou, G. 1992, MNRAS, 256, 43P

Eke, V. R., Cole, S., \& Frenk, C. S. 1996, MNRAS, 282, 263

Frebel, A. 2005, Nature, 434, 871

Gao, L., \& White, S. D. M. 2006, MNRAS, 373, 65

Garrison-Kimmel, S., Rocha, M., Boylan-Kolchin, M., Bullock, J. S., \& Lally, J. 2013, MNRAS, 433, 3539

Green, A. M., Hofmann, S., \& Schwarz, D. J. 2004, MNRAS, 353, L23

Hahn, O., Abel, T., \& Kaehler, R. 2013, MNRAS, 434, 1171

Klypin, A., Holtzman, J., Primack, J., \& Regos, E. 1993, ApJ, 416, 1

Knollmann, S. R., \& Knebe, A. 2009, ApJS, 182, 608

Loeb, A., \& Weiner, N. 2011, PhRvL, 106, 171302

Łokas, E. L., \& Mamon, G. A. 2001, MNRAS, 321, 155

Macciò, A. V., Paduroiu, S., Anderhalden, D., Schneider, A., \& Moore, B. 2012, MNRAS, 424, 1105

Maulbetsch, C., Avila-Reese, V., Colín, P., Gottlöber, S., Khalatyan, A., \& Steinmetz, M. 2007, ApJ, 654, 53

Menci, N., Fiore, F., \& Lamastra, A. 2012, MNRAS, 2425

Moore, B. 1994, Natur, 370, 629

Moore, B., Quinn, T., Governato, F., Stadel, J., \& Lake, G. 1999, MNRAS, 310, 1147

Murray, S., Power, C., \& Robotham, A. 2013a, arxiv:1306.6721

Murray, S. G., Power, C., \& Robotham, A. S. G. 2013b, MNRAS, 434, L61

Pacucci, F., Mesinger, A., \& Haiman, Z. 2013, MNRAS, 435, L53

Peebles, P. J. E. 1969, ApJ, 155, 393

Peter, A. H. G., Rocha, M., Bullock, J. S., \& Kaplinghat, M. 2013, MNRAS, 430, 105

Planck Collaboration, et al. 2013, arxiv:1303.5076
Power, C., Knebe, A., \& Knollmann, S. R. 2012, MNRAS, 419, 1576

Power, C., Navarro, J. F., Jenkins, A., Frenk, C. S., White, S. D. M., Springel, V., Stadel, J., \& Quinn, T. 2003, MNRAS, 338, 14

Prada, F., Klypin, A. A., Simonneau, E., Betancort-Rijo, J., Patiri, S., Gottlöber, S., \& Sanchez-Conde, M. A. 2006, ApJ, 645, 1001

Primack, J. R. 2009, in American Institute of Physics Conference Series, Vol. 1166, ed. D. B. Cline, 3, 1192, 101

Rocha, M., Peter, A. H. G., Bullock, J. S., Kaplinghat, M., GarrisonKimmel, S., Oñorbe, J., \& Moustakas, L. A. 2013, MNRAS, 430, 81

Schneider, A., Smith, R. E., Maccio, A. V., \& Moore, B. 2012, MNRAS, 424, 684

Schneider, A., Smith, R. E., \& Reed, D. 2013, MNRAS, 433, 1573

Seljak, U., \& Zaldarriaga, M. 1996, ApJ, 469, 437

Shaw, L. D., Weller, J., Ostriker, J. P., \& Bode, P. 2006, ApJ, 646, 815

Smith, R. E., \& Markovic, K. 2011, PhRvD, 84, 063507

Spergel, D. N. 2007, ApJS, 170, 377

Springel, V. 2005, MNRAS, 364, 1105

Springel, V., et al. 2008, MNRAS, 391, 1685

Sugerman, B., Summers, F. J., \& Kamionkowski, M. 2000, MNRAS, 311, 762

Thoul, A. A., \& Weinberg, D. H. 1996, ApJ, 465, 608

Tremaine, S., \& Gunn, J. E. 1979, PhRvL, 42, 407

Villaescusa-Navarro, F., \& Dalal, N. 2011, JCAP, 3, 24

Vogelsberger, M., Zavala, J., \& Loeb, A. 2012, MNRAS, 3127

Wang, J., \& White, S. D. M. 2007, MNRAS, 380, 93

Wang, J., \& White, S. D. M. 2009, MNRAS, 396, 709

White, S. D. M. 1984, ApJ, 286, 38

White, S. D. M. 1994, arXiv:astro-ph/9410043

White, S. D. M., \& Frenk, C. S. 1991, ApJ, 379, 52

White, S. D. M., \& Rees, M. J. 1978, MNRAS, 183, 341

Yoshida, N., Springel, V., White, S. D. M., \& Tormen, G. 2000, ApJ, 544, L87 